\title{
Mean zonal flows generated by librations of a rotating spherical cavity
}

\author{
F. H. BUSSE† \\ Institute of Physics, University of Bayreuth, Bayreuth 95440, Germany \\ (Received 30 November 2009; revised 4 February 2010; accepted 5 February 2010; \\ first published online 19 March 2010)
}

Longitudinal librations represent oscillations about the axis of a rotating axisymmetric fluid-filled cavity. An analytical theory is developed for the case of a spherical cavity in the limit when the libration frequency is small in comparison with the rotation rate, but large in comparison with the inverse of the spin-up time. It is shown that longitudinal librations create a steady zonal flow through the nonlinear advection in the Ekman layers. The theory can be applied to laboratory experiments as well as to solid planets and satellites with a liquid core.

\section{Introduction}

Longitudinal librations are of considerable interest in planetology since they represent a major dynamical response of rotating solid planets and moons to an applied gravitational field. Because these bodies are usually not entirely axisymmetric and their orbits about a central source of gravitation exhibits a finite excentricity, axial torques of alternating signs will be exerted on these bodies. Since the latter often posses a liquid core which is assumed to be axisymmetric, the oscillations of the core boundary are transmitted to the liquid only through thin viscous boundary layers called Ekman layers. Because of the usually low viscosity of the liquid the spin-up time of the core is typically much longer than the period of the applied torque with the consequence that the liquid core only slightly participates in the librations of the solid mantle. There is the possibility, however, that inertial modes are excited at certain frequencies of libration as has been demonstrated in the experiments of Aldridge \& Toomre (1969). This subject will not be addressed in the analysis of this paper in which the limit of low libration frequencies will be considered. In this limit the possibility of a geostrophic response generated through nonlinear effects of motions in the Ekman layers is of primary interest. This phenomenon will be described by an analytical theory in the weakly nonlinear limit.

Only longitudinal librations will be studied, i.e. small sinusoidal variation of the rotation of a spherical cavity about a fixed axis. For simplicity the attribute 'longitudinal' will be dropped in the following. The paper starts with the mathematical formulation of the problem in $\S 2$. In $\S 3$ the linear analysis is described. Weakly nonlinear results are presented in $\S 4$. Concluding remarks and a discussion of applications are offered in $\S 5$.

\section{Mathematical formulation}

We consider a fluid-filled spherical cavity of radius $R$ that is rotating in the time average with the constant angular velocity $\Omega$. Using $R$ as length scale and $1 / \Omega$ as

$\dagger$ Email address for correspondence: Busse@uni-bayreuth.de 
time scale we can write the equations of motion in dimensionless form,

$$
\begin{aligned}
\partial_{t} \boldsymbol{u}+\boldsymbol{u} \cdot \nabla \boldsymbol{u}+2 \boldsymbol{k} \times \boldsymbol{u} & =-\nabla \pi+E \nabla^{2} \boldsymbol{u}, \\
\nabla \cdot \boldsymbol{u} & =0,
\end{aligned}
$$

where $\boldsymbol{k}$ is the unit vector in the direction of the axis of rotation and the Ekman number $E$ is defined by

$$
E=v / \Omega R^{2}
$$

Here $v$ denotes the kinematic viscosity of the fluid. The boundary condition is given by

$$
\boldsymbol{u}=\boldsymbol{k} \times \boldsymbol{r} \in \cos \omega t \quad \text { at } \quad|\boldsymbol{r}|=1,
$$

where $\boldsymbol{r}$ is the position vector with respect to the centre of the sphere and $\omega$ is the angular frequency of libration.

In order to solve the problem posed by (2.1) and (2.3) in the limit of small values of $E$ we separate the velocity field $\boldsymbol{u}$ into two parts, the component $\boldsymbol{U}$ describing the flow in the interior of the spherical cavity where viscous friction is negligible and the boundary layer component $\tilde{\boldsymbol{u}}$ which assumes finite values in the Ekman layer near the solid surface $|\boldsymbol{r}|=1$, but decays exponentially towards the interior. The equations for the two components are thus given by

$$
\begin{aligned}
\partial_{t} \boldsymbol{U}+\boldsymbol{U} \cdot \nabla \boldsymbol{U}+2 \boldsymbol{k} \times \boldsymbol{U} & =-\nabla \pi, \\
\nabla \cdot \boldsymbol{U}=0, \quad \nabla \cdot \tilde{\boldsymbol{u}} & =0, \\
\partial_{t} \tilde{\boldsymbol{u}}+(\boldsymbol{U}+\tilde{\boldsymbol{u}}) \cdot \nabla \tilde{\boldsymbol{u}}+\tilde{\boldsymbol{u}} \cdot \nabla \boldsymbol{U}+2 \boldsymbol{k} \times \tilde{\boldsymbol{u}} & =-\nabla \pi+E \nabla^{2} \tilde{\boldsymbol{u}} .
\end{aligned}
$$

Because the Ekman layer thickness is in general of the order $\sqrt{E}$, the radial derivative in the Laplace operator of $(2.4 c)$ by far exceeds the derivatives parallel to the boundary. It is thus appropriate to introduce $\xi=(1-r) / \sqrt{E}$ as variable. Introducing the ansatz

$$
\tilde{\boldsymbol{u}}=\epsilon\left(\hat{\boldsymbol{u}}_{1} \cos \omega t+\check{\boldsymbol{u}}_{1} \sin \omega t\right)
$$

we obtain as linearized version of $(2.4 c)$ :

$$
\begin{gathered}
\omega \check{\boldsymbol{u}}_{1}+2 \boldsymbol{k} \times \hat{\boldsymbol{u}}_{1}=\frac{\partial^{2}}{\partial \xi^{2}} \hat{\boldsymbol{u}}_{1}, \\
-\omega \hat{\boldsymbol{u}}_{1}+2 \boldsymbol{k} \times \check{\boldsymbol{u}}_{1}=\frac{\partial^{2}}{\partial \xi^{2}} \check{\boldsymbol{u}}_{1},
\end{gathered}
$$

where in addition to the nonlinear terms the pressure gradient has been dropped according to the boundary layer assumption. By operating with $\boldsymbol{n} \times$ and $i \boldsymbol{n} \times(\boldsymbol{n} \times \ldots)$ onto each of the equations $(2.6 a, b)$ where $\boldsymbol{n}$ is the normal unit vector of the boundary we obtain

$$
\begin{array}{r}
\omega\left(\boldsymbol{n} \times \check{\boldsymbol{u}}_{1}+i \check{\boldsymbol{u}}_{1}\right)+2 i \boldsymbol{k} \cdot \boldsymbol{n}\left(\boldsymbol{n} \times \hat{\boldsymbol{u}}_{1}+i \hat{\boldsymbol{u}}_{1}\right)=\frac{\partial^{2}}{\partial \xi^{2}}\left(\boldsymbol{n} \times \hat{\boldsymbol{u}}_{1}+i \hat{\boldsymbol{u}}_{1}\right), \\
-\omega\left(\boldsymbol{n} \times \hat{\boldsymbol{u}}_{1}+i \hat{\boldsymbol{u}}_{1}\right)+2 i \boldsymbol{k} \cdot \boldsymbol{n}\left(\boldsymbol{n} \times \check{\boldsymbol{u}}_{1}+i \check{\boldsymbol{u}}_{1}\right)=\frac{\partial^{2}}{\partial \xi^{2}}\left(\boldsymbol{n} \times \check{\boldsymbol{u}}_{1}+i \check{\boldsymbol{u}}_{1}\right),
\end{array}
$$

where the normal component of the velocity has been neglected since it is of the order $\sqrt{E}$ smaller than the tangential components. Equations (2.7) together with the 
boundary condition (2.3) are solved by

$$
\begin{aligned}
& \boldsymbol{n} \times \hat{\boldsymbol{u}}_{1}+i \hat{\boldsymbol{u}}_{1}=\frac{1}{2}(\boldsymbol{n} \times(\boldsymbol{k} \times \boldsymbol{r})+i \boldsymbol{k} \times \boldsymbol{r})\left(\exp \left\{\lambda_{+} \xi\right\}+\exp \left\{\lambda_{-} \xi\right\}\right), \\
& \boldsymbol{n} \times \check{\boldsymbol{u}}_{1}+i \check{\boldsymbol{u}}_{1}=\frac{i}{2}(\boldsymbol{n} \times(\boldsymbol{k} \times \boldsymbol{r})+i \boldsymbol{k} \times \boldsymbol{r})\left(\exp \left\{\lambda_{+} \xi\right\}-\exp \left\{\lambda_{-} \xi\right\}\right),
\end{aligned}
$$

where the coefficients $\lambda_{ \pm}$are the roots of the equation

$$
\lambda_{ \pm}^{2}-2 i \boldsymbol{k} \cdot \boldsymbol{n}= \pm i \omega .
$$

Since roots with positive real parts satisfy the condition of decay towards the interior, we find

$$
\lambda_{ \pm}=-\left(1+i s_{ \pm}\right) \sqrt{|\boldsymbol{k} \cdot \boldsymbol{n} \pm \omega / 2|}
$$

where $s_{ \pm}$denotes the sign of $\boldsymbol{k} \cdot \boldsymbol{n} \pm \omega / 2$.

Since the spin-up time of the interior is of the order $1 / \sqrt{E}$ (Greenspan 1968), hardly any spin-up occurs according to the linear theory as long as $\omega \gg \sqrt{E}$ holds. The nonlinear terms of the equations of motion may give rise to a steady component of the velocity field in the boundary layer and thus may induce a finite flow in the interior. In order to study this situation we shall assume that $\epsilon \ll 1$ holds such that a weakly nonlinear analysis can be applied. To simplify the problem further we shall focus on the limit of small $\omega$ such that the inequalities

$$
\sqrt{E} \ll \omega \ll \epsilon \ll 1
$$

hold. The assumption of the limit of small $\omega$ allows us to neglect in first approximation the $\check{\boldsymbol{u}}_{1}$-component of the velocity field and to rewrite the $\hat{\boldsymbol{u}}_{1}$-component in the form

$$
\boldsymbol{n} \times \hat{\boldsymbol{u}}_{1}+i \hat{\boldsymbol{u}}_{1}=(\boldsymbol{n} \times(\boldsymbol{k} \times \boldsymbol{r})+i \boldsymbol{k} \times \boldsymbol{r}) \exp \{\lambda \xi\}
$$

with

$$
\lambda=-(1+i s) \sqrt{|\boldsymbol{k} \cdot \boldsymbol{n}|},
$$

where $s= \pm 1$ denotes the sign of $\boldsymbol{k} \cdot \boldsymbol{n}$.

\section{Weakly nonlinear analysis}

For the weakly nonlinear analysis we use the small parameter $\epsilon$ as expansion parameter. Among the contributions to the velocity field of the order $\epsilon^{2}$ the timeindependent part $\epsilon^{2} \overline{\boldsymbol{u}}_{2}$ of $\tilde{\boldsymbol{u}}$ is of primary interest. It is governed by the equations

$$
\begin{aligned}
& \nabla \cdot \overline{\boldsymbol{u}}_{2}=0, \\
& \hat{\boldsymbol{u}}_{1} \cdot \nabla \hat{\boldsymbol{u}}_{1} / 2-\epsilon^{-2} \overline{(\boldsymbol{U}+\tilde{\boldsymbol{u}}) \cdot \boldsymbol{n} \frac{\partial}{\partial \xi} \tilde{\boldsymbol{u}} / \sqrt{E}}+2 \boldsymbol{k} \times \overline{\boldsymbol{u}}_{2}=\frac{\partial^{2}}{\partial \xi^{2}} \overline{\boldsymbol{u}}_{2},
\end{aligned}
$$

where the bar indicates the time average and where it has been used that only the normal component of the interior flow $\boldsymbol{U}$ contributes in this equation since it is associated with the normal derivative which is of the order $1 / \sqrt{E}$. The normal velocity close to the boundary, $\boldsymbol{n} \cdot(\boldsymbol{U}+\tilde{\boldsymbol{u}})$, is given by the eflux from the Ekman layer, which in first approximation is determined by

$$
\frac{\partial}{\partial \xi} \boldsymbol{n} \cdot \tilde{\boldsymbol{u}}=\epsilon \sqrt{E} \boldsymbol{n} \cdot \nabla \times\left(\boldsymbol{n} \times \hat{\boldsymbol{u}}_{1}\right) \cos \omega t .
$$


As solution of this equation together with the boundary condition $\boldsymbol{n} \cdot(\boldsymbol{U}+\tilde{\boldsymbol{u}})=0$ at $\xi=0$ we obtain

$$
\begin{aligned}
\boldsymbol{n} \cdot \tilde{\boldsymbol{u}}= & \epsilon \sqrt{E} \cos \omega t\left(\boldsymbol{n} \cdot \boldsymbol{k i}\left(\lambda^{-1} \exp \{\lambda \xi\}-\lambda^{*-1} \exp \left\{\lambda^{*} \xi\right\}\right)+|\boldsymbol{n} \times \boldsymbol{k}|^{2}\right. \\
& \left.\cdot\left(\left(\xi \lambda^{-2}-\lambda^{-3}\right) \exp \{\lambda \xi\}+\left(\xi \lambda^{*-2}-\lambda^{*-3}\right) \exp \left\{\lambda^{*} \xi\right\}\right) / 2\right), \\
\boldsymbol{n} \cdot \boldsymbol{U}= & -\epsilon \sqrt{E} \cos \omega t\left(\boldsymbol{n} \cdot \boldsymbol{k} i\left(\lambda^{-1}-\lambda^{*-1}\right)-\frac{1}{2}\left(1-(\boldsymbol{n} \cdot \boldsymbol{k})^{2}\right)\left(\lambda^{-3}+\lambda^{*-3}\right)\right),
\end{aligned}
$$

where $\lambda^{*}$ indicates the complex conjugate of $\lambda$.

As Greenspan \& Weinbaum (1965) have pointed out, expansions of the type we are using here are not uniformly valid. For this reason we shall include the term $\epsilon^{-2} \boldsymbol{U} \cdot \boldsymbol{n} \frac{\partial}{\partial \xi} \tilde{\boldsymbol{u}} / \sqrt{E}$ of $(3.1 b)$ already in $(2.7 a)$. The latter equation thus assumes the form

$$
2 i \boldsymbol{k} \cdot \boldsymbol{n}\left(\boldsymbol{n} \times \hat{\hat{\boldsymbol{u}}}_{1}+i \hat{\boldsymbol{u}}_{1}\right)-\frac{\boldsymbol{n} \cdot \boldsymbol{U}}{\sqrt{E}} \frac{\partial}{\partial \xi}\left(\boldsymbol{n} \times \hat{\hat{\boldsymbol{u}}}_{1}+i \hat{\boldsymbol{u}}_{1}\right)=\frac{\partial^{2}}{\partial \xi^{2}}\left(\boldsymbol{n} \times \hat{\boldsymbol{u}}_{1}+i \hat{\hat{\boldsymbol{u}}}_{1}\right),
$$

the solution of which is given by

$$
\begin{aligned}
\boldsymbol{n} \times \hat{\boldsymbol{u}}_{1}+i \hat{\boldsymbol{\mu}}_{1}=(\boldsymbol{n} \times(\boldsymbol{k} \times \boldsymbol{r})+i \boldsymbol{k} \times \boldsymbol{r}) \\
\cdot \exp \left\{\lambda \xi\left(\sqrt{1+(\boldsymbol{n} \cdot \boldsymbol{U} / 2 \sqrt{E} \lambda)^{2}}-\boldsymbol{n} \cdot \boldsymbol{U} / 2 \sqrt{E} \lambda\right)\right\} .
\end{aligned}
$$

According to this expression the solution $\hat{\hat{\boldsymbol{u}}}$ includes a term of the order $\epsilon \cos \omega t$ in addition to $\hat{\boldsymbol{u}}_{1}$ as given by expression (2.12):

$$
\begin{array}{r}
\boldsymbol{n} \times \hat{\boldsymbol{\boldsymbol { u }}}_{1}+i \hat{\boldsymbol{\boldsymbol { u }}}_{1}=\boldsymbol{n} \times\left(\hat{\boldsymbol{u}}_{1}+2 \epsilon \overline{\boldsymbol{u}}_{2}^{A} \cos \omega t\right)+i\left(\hat{\boldsymbol{u}}_{1}+2 \epsilon \overline{\boldsymbol{u}}_{2}^{A} \cos \omega t\right)=(\boldsymbol{n} \times(\boldsymbol{k} \times \boldsymbol{r})+i \boldsymbol{k} \times \boldsymbol{r}) \\
\cdot \exp \{\lambda \xi\}\left(1+\xi \epsilon \cos \omega t\left(\frac{\boldsymbol{n} \cdot \boldsymbol{k} i}{2 \lambda}\left(1-\frac{\lambda}{\lambda^{*}}\right)-|\boldsymbol{n} \times \boldsymbol{k}|^{2}\left(\lambda^{-3}+\lambda^{*-3}\right) / 4\right)\right), \quad \text { (3.7) }
\end{array}
$$

where terms of the order $\epsilon^{2}$ have been neglected. Since we have dropped the timedependence $\epsilon \cos \omega t$ in formulating (3.5a), we conclude that $\epsilon^{2} \overline{\boldsymbol{u}}_{2}^{A}$ given by

$$
\boldsymbol{n} \times \overline{\boldsymbol{u}}_{2}^{A}=\boldsymbol{k} \times \boldsymbol{r}\left(\left(\exp \{\lambda \xi\}-\exp \left\{\lambda^{*} \xi\right\}\right) \xi \frac{1}{8 i} \sqrt{|\boldsymbol{k} \cdot \boldsymbol{n}|}\left(1+\frac{|\boldsymbol{n} \times \boldsymbol{k}|^{2}}{4|\boldsymbol{k} \cdot \boldsymbol{n}|^{2}}\right)\right)+\boldsymbol{n} \times(\boldsymbol{k} \times \boldsymbol{r})(\ldots)
$$

represents a first contribution to the time-independent part $\epsilon^{2} \overline{\boldsymbol{u}}_{2}$ of $\tilde{\boldsymbol{u}}$. The term proportional to $\boldsymbol{n} \times(\boldsymbol{k} \times \boldsymbol{r})$ has not been given explicitly in expression (3.8) since it will not be needed in the following.

Without the term with $\boldsymbol{n} \cdot \boldsymbol{U}$ we are now able to solve equations (3.1). Introducing $j$ as new imaginary unit we write

$$
\left(\frac{\partial^{2}}{\partial \xi^{2}}-j 2 \boldsymbol{k} \cdot \boldsymbol{n}\right)\left(\boldsymbol{n} \times \overline{\boldsymbol{u}}_{2}+j \overline{\boldsymbol{u}}_{2}\right)=(\boldsymbol{n} \times-j \boldsymbol{n} \times(\boldsymbol{n} \times \ldots))\left(\hat{\boldsymbol{u}}_{1} \cdot \nabla \hat{\boldsymbol{u}}_{1}-\hat{u}_{n} \frac{\partial}{\partial \xi} \hat{\boldsymbol{u}}_{1}\right) / 2,
$$

where the abbreviation $\hat{u}_{n} \cos \omega t=\boldsymbol{n} \cdot \tilde{\boldsymbol{u}} / \epsilon$ has been used. The solution $\overline{\boldsymbol{u}}_{2}^{B}$ of this equation satisfying the boundary condition $\overline{\boldsymbol{u}}_{2}^{B}=0$ at $\xi=0$ is derived in the Appendix. The two imaginary units $i$ and $j$ are entirely independent and they have been introduced only in order to obtain a compact notation.

The general solution $\bar{U}$ of $(2.4 a)$ describing steady flow in the interior and satisfying the boundary condition $\boldsymbol{n} \cdot \overline{\boldsymbol{U}}=0$ is given by

$$
\overline{\boldsymbol{U}}=\epsilon^{2} \boldsymbol{k} \times \boldsymbol{r} f\left(|\boldsymbol{k} \times \boldsymbol{r}|^{2}\right),
$$


according to the Taylor-Proudman theorem (Greenspan 1968) where we have anticipated that this axisymmetric zonal flow will be of the order $\epsilon^{2}$. Connected with this differential rotation is an Ekman layer flow $\epsilon^{2} \overline{\boldsymbol{u}}_{2}^{C}$ which is determined by

$$
\boldsymbol{n} \times \overline{\boldsymbol{u}}_{2}^{C}+i \overline{\boldsymbol{u}}_{2}^{C}=-(\boldsymbol{n} \times(\boldsymbol{k} \times \boldsymbol{r})+i \boldsymbol{k} \times \boldsymbol{r}) f\left(|\boldsymbol{k} \times \boldsymbol{r}|^{2}\right) \exp \{\lambda \xi\} .
$$

Since all three contributions $\overline{\boldsymbol{u}}_{2}^{A}, \overline{\boldsymbol{u}}_{2}^{B}$ and $\overline{\boldsymbol{u}}_{2}^{C}$ are symmetric with respect to the equatorial plane the corresponding suctions induced by these steady Ekman layer flows are antisymmetric with respect to this plane and thus must vanish in the case of a steady state,

$$
\boldsymbol{n} \cdot \nabla \times \int_{0}^{\infty} \boldsymbol{n} \times\left(\overline{\boldsymbol{u}}_{2}^{A}+\overline{\boldsymbol{u}}_{2}^{B}+\overline{\boldsymbol{u}}_{2}^{C}\right) \mathrm{d} \xi=0,
$$

since otherwise a steady differential rotation of the form (3.10) will not be possible. Condition (3.12) thus determines the so far unknown function $f\left(|\boldsymbol{k} \times \boldsymbol{r}|^{2}\right)$.

\section{The mean zonal flow}

For the evaluation of condition (3.12) we first consider the simpler term involving $\overline{\boldsymbol{u}}_{2}^{C}$. The component proportional to $\boldsymbol{n} \times(\boldsymbol{k} \times \boldsymbol{r})$ of $\boldsymbol{n} \times \overline{\boldsymbol{u}}_{2}^{C}$ does not contribute to the left-hand side of (3.10). Accordingly we obtain

$$
\boldsymbol{n} \cdot \nabla \times \int_{0}^{\infty} \boldsymbol{n} \times \overline{\boldsymbol{u}}_{2}^{C} \mathrm{~d} \xi=-\sqrt{\boldsymbol{n} \cdot \boldsymbol{k}}\left(f\left(x^{2}\right)\left(1+\frac{x^{2}}{4\left(1-x^{2}\right)}\right)+x^{2} f^{\prime}\left(x^{2}\right)\right),
$$

where $x \equiv|\boldsymbol{k} \times \boldsymbol{n}|$ has been introduced as an abbreviation.

Using the expression

$$
\boldsymbol{n} \cdot \nabla \times \int_{0}^{\infty} \boldsymbol{n} \times \overline{\boldsymbol{u}}_{2}^{A} \mathrm{~d} \xi=-\sqrt{\boldsymbol{n} \cdot \boldsymbol{k}}\left(1+\frac{|\boldsymbol{n} \times \boldsymbol{k}|^{2}}{2|\boldsymbol{k} \cdot \boldsymbol{n}|^{2}}+\frac{|\boldsymbol{n} \times \boldsymbol{k}|^{4}}{16|\boldsymbol{k} \cdot \boldsymbol{n}|^{4}}+\frac{|\boldsymbol{n} \times \boldsymbol{k}|^{2}}{4|\boldsymbol{k} \cdot \boldsymbol{n}|^{4}}\right) / 4
$$

and expression (A 7) from the Appendix, condition (3.12) can now be written in the form of a differential equation for $f\left(x^{2}\right)$,

$f^{\prime}\left(x^{2}\right)=-f\left(x^{2}\right)\left(1+\frac{x^{2}}{4\left(1-x^{2}\right)}\right) / x^{2}-\left(\frac{360}{x^{2}}+\frac{663}{4\left(1-x^{2}\right)}+\frac{505}{4\left(1-x^{2}\right)^{2}}\right) / 2400$,

where the prime indicates the differentiation with respect to $x^{2}$. Equation (4.3) has the solution

$$
f\left(x^{2}\right)=\frac{259 x^{2}-360}{2400\left(1-x^{2}\right)}
$$

where the constant of integration vanishes since it is determined by the condition that $f\left(x^{2}\right)$ is finite at $x=0$. Because only one constant of integration exists the singularity at $x=1$ can not be avoided. Such a singularity must expected in any case since the Ekman boundary layer diverges at $x=1$.

The function $f\left(x^{2}\right)$ has been plotted in figure 1. Because of its negative sign the fluid rotates in a retrograde fashion relative to the librating spherical cavity. Since the absolute value of the angular momentum decreases with increasing $x$ near the equator a centrifugal instability in the form of small axisymmetric vortices can be expected according to Rayleigh's criterion. The divergence of $f\left(x^{2}\right)$ and of the Ekman layer thickness at the equator requires the introduction of new scalings (see e.g. Stewartson \& Roberts 1963). But it is to be expected that the mean zonal flow will be affected only close to the equator in the limit $E \rightarrow 0$. 


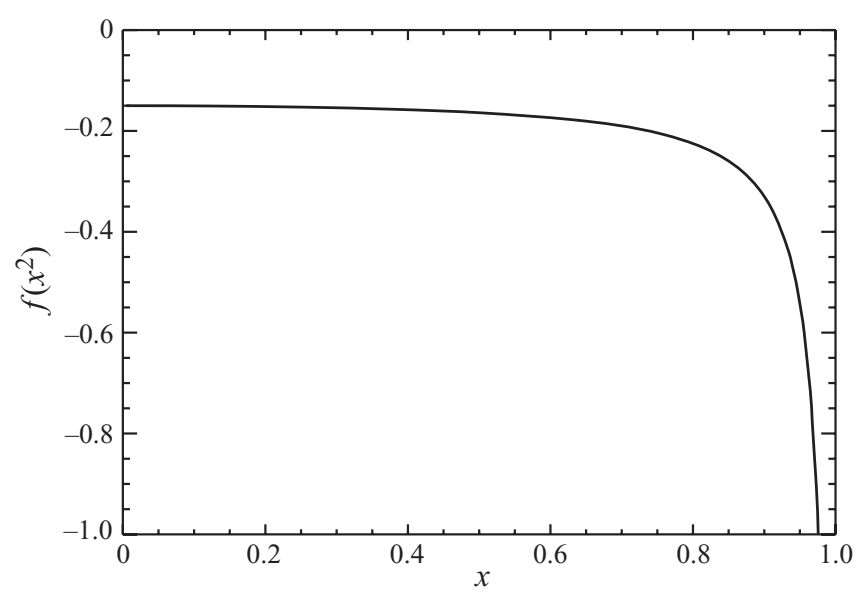

Figure 1. The function $f\left(x^{2}\right)=f\left(|\boldsymbol{k} \times \boldsymbol{r}|^{2}\right)$ in dependence on $x \equiv|\boldsymbol{k} \times \boldsymbol{r}|$.

\section{Discussion}

There do not seem to exist in the literature any measurements of the differential rotation induced by low-frequency librations. In the reports on experimental observations the occurrence of Taylor-Görtler type axisymmetric vortices is mentioned (Aldridge 1967; Noir et al. 2009), but they are attributed to the centrifugal instability during the retrograde phase of the libration. When this instability is growing only weakly, however, positive growth rates during the retrograde phase are compensated by corresponding negative growth rates during the prograde phase of librations. In this case the unstable profile of the mean flow found in the analysis of $\S 4$ may be essential for the generation of axisymmetric vortices even though it is only of the order $\epsilon^{2}$, and thus small compared to the amplitude $\epsilon$ of the libration.

Because the differential rotation is essentially constant up to a distance of nearly $x=0.6$ from the axis of the cavity, the solution derived in the foregoing sections will be affected only very little by the presence of a concentric solid spherical inner core with a radius $x \lesssim 0.6$. The mean rotation rate of such an inner core will be slightly less, of course, than that of the outer boundary.

For a discussion of planetary application we refer to the paper of Noir et al. (2009). In this paper a distinction is made between molten cores such as those of Earth's moon and of Ganymede and of the subsurface ocean of Callisto and those cores where the Reynolds number based on the Ekman flow is sufficiently large such that a turbulent response must be expected. The latter situation would apply to the molten cores of Io and Mercury and the subsurface oceans on Titan and Europa. Of particular interest is the phenomenon of seasonal librations caused by the shifting wind systems in an atmosphere above a solid mantle. In the case of Earth this effect appears to be quite weak (Wahr 1988), but a much stronger seasonal torque can be expected in the case of Titan (Van Hoolst, Rambaux \& Karatekin 2009) which may give rise to turbulence in its subsurface oceanic layer as mentioned above.

The author gratefully acknowledges the support he received from the Spanish government through contract FIS2008-01126 and from NORDITA during a stay in Stockholm. A discussion with Michael Calkins and Jerome Noir on problems of libration has stimulated the author to find a mistake in the original version of the paper. 


\section{Appendix. Boundary layer solution}

Using

$$
\hat{\boldsymbol{u}}_{1}=\boldsymbol{n} \times(\boldsymbol{k} \times \boldsymbol{r})\left(\exp \{\lambda \xi\}-\exp \left\{\lambda^{*} \xi\right\}\right) / 2 i+\boldsymbol{k} \times \boldsymbol{r}\left(\exp \{\lambda \xi\}+\exp \left\{\lambda^{*} \xi\right\}\right) / 2,
$$

we find

$$
\begin{aligned}
\hat{\boldsymbol{u}}_{1} \cdot \nabla \hat{\boldsymbol{u}}_{1}= & \boldsymbol{n} \times(\boldsymbol{k} \times \boldsymbol{r})\left(\boldsymbol{k} \cdot \boldsymbol{n}\left(\exp \{\lambda \xi\}-\exp \left\{\lambda^{*} \xi\right\}\right)^{2}-i \xi|\boldsymbol{n} \times \boldsymbol{k}|^{2}(\exp \{\lambda \xi\}\right. \\
& \left.\left.-\exp \left\{\lambda^{*} \xi\right\}\right)\left(\lambda^{-1} \exp \{\lambda \xi\}+\lambda^{*-1} \exp \left\{\lambda^{*} \xi\right\}\right)\right) / 4+\boldsymbol{k} \times \boldsymbol{r}(2 i(\exp \{2 \lambda \xi\} \\
& \left.-\exp \left\{2 \lambda^{*} \xi\right\}\right) \boldsymbol{n} \cdot \boldsymbol{k}+\xi|\boldsymbol{n} \times \boldsymbol{k}|^{2}\left(\exp \{\lambda \xi\}-\exp \left\{\lambda^{*} \xi\right\}\right)\left(\lambda^{-1} \exp \{\lambda \xi\}\right. \\
& \left.\left.-\lambda^{*-1} \exp \left\{\lambda^{*} \xi\right\}\right)\right) / 4+\boldsymbol{k} \times(\boldsymbol{k} \times \boldsymbol{r})\left(\exp \{\lambda \xi\}+\exp \left\{\lambda^{*} \xi\right\}\right)^{2} / 4 .
\end{aligned}
$$

Since $\hat{u}_{n}$ is determined by

$$
\frac{\partial}{\partial \xi} \hat{u}_{n}=\boldsymbol{k} \cdot \boldsymbol{n i}\left(\exp \{\lambda \xi\}-\exp \left\{\lambda^{*} \xi\right\}\right)+|\boldsymbol{k} \times \boldsymbol{n}|^{2} \xi\left(\exp \{\lambda \xi\} \lambda^{-1}+\exp \left\{\lambda^{*} \xi\right\} \lambda^{*-1}\right) / 2,
$$

we find that

$$
\begin{aligned}
-\hat{u}_{n} \frac{\partial}{\partial \xi} \hat{\boldsymbol{u}}_{1}= & \boldsymbol{n} \times(\boldsymbol{k} \times \boldsymbol{r})\left(-\boldsymbol{k} \cdot \boldsymbol{n}\left(\exp \{2 \lambda \xi\}+\exp \left\{2 \lambda^{*} \xi\right\}-\left(\frac{\lambda}{\lambda^{*}}+\frac{\lambda^{*}}{\lambda}\right)\right.\right. \\
& \left.\times \exp \left\{\left(\lambda+\lambda^{*}\right) \xi\right\}\right) / 2+\frac{i}{4}|\boldsymbol{n} \times \boldsymbol{k}|^{2}\left(\frac{\xi \lambda-1}{\lambda^{2}} \exp \{2 \lambda \xi\}-\frac{\xi \lambda^{*}-1}{\lambda^{* 2}} \exp \left\{2 \lambda^{*} \xi\right\}\right. \\
& \left.\left.-\left(\lambda^{*} \frac{\xi \lambda-1}{\lambda^{3}}-\lambda \frac{\xi \lambda^{*}-1}{\lambda^{* 3}}\right) \exp \left\{\left(\lambda+\lambda^{*}\right) \xi\right\}\right)\right)+\boldsymbol{k} \times \boldsymbol{r}(-i(\exp \{2 \lambda \xi\} \\
& -\exp \left\{2 \lambda^{*} \xi-\left(\frac{\lambda}{\lambda^{*}}-\frac{\lambda^{*}}{\lambda}\right) \exp \left\{\left(\lambda+\lambda^{*}\right) \xi\right\}\right) \boldsymbol{n} \cdot \boldsymbol{k} / 2-\frac{|\boldsymbol{n} \times \boldsymbol{k}|^{2}}{4}\left(\frac{\xi \lambda-1}{\lambda^{2}} \exp \{2 \lambda \xi\}\right. \\
& \left.\left.+\frac{\xi \lambda^{*}-1}{\lambda^{* 2}} \exp \left\{2 \lambda^{*} \xi\right\}+\frac{\lambda^{* 4}(\xi \lambda-1)+\lambda^{4}\left(\xi \lambda^{*}-1\right)}{\lambda^{* 3} \lambda^{3}} \exp \left\{\left(\lambda+\lambda^{*}\right) \xi\right\}\right)\right) .
\end{aligned}
$$

Fortunately a number of terms cancel when the expressions (A 2) and (A 4) are added. Further simplifications result from relationships such as $\left(\lambda / \lambda^{*}\right)+\left(\lambda^{*} / \lambda\right)=0,\left(\lambda / \lambda^{* 3}\right)-$ $\left(\lambda^{*} / \lambda^{3}\right)=0$. The solution $\overline{\boldsymbol{u}}_{2}^{B}$ of $(3.7)$ can thus be obtained in the form

$$
\begin{aligned}
\boldsymbol{n} \times \overline{\boldsymbol{u}}_{2}^{B}+j \overline{\boldsymbol{u}}_{2}^{B}= & (\boldsymbol{n} \times(\boldsymbol{k} \times \boldsymbol{r})+j \boldsymbol{k} \times \boldsymbol{r})\left(-\frac{1}{4(2-j s)}\left(\exp \left\{\left(\lambda+\lambda^{*}\right) \xi\right\}-\exp \{\kappa \xi\}\right)\right. \\
& +|\boldsymbol{n} \times \boldsymbol{k}|^{2}\left(\frac{\exp \{2 \lambda \xi\}-\exp \{\kappa \xi\}}{16 i|\boldsymbol{k} \cdot \boldsymbol{n}|^{2}(4 i-j)}-\frac{\exp \left\{2 \lambda^{*} \xi\right\}-\exp \{\kappa \xi\}}{16 i|\boldsymbol{k} \cdot \boldsymbol{n}|^{2}(-4 i-j)}\right. \\
& \left.\left.+\frac{\exp \left\{\left(\lambda+\lambda^{*}\right) \xi\right\}-\exp \{\kappa \xi\}}{16 s|\boldsymbol{k} \cdot \boldsymbol{n}|^{2}(2 s-j)}\right)\right)+(j \boldsymbol{n} \times(\boldsymbol{k} \times \boldsymbol{r})-\boldsymbol{k} \times \boldsymbol{r}) \frac{|\boldsymbol{n} \times \boldsymbol{k}|^{2}}{16|\boldsymbol{k} \cdot \boldsymbol{n}|^{2}} \\
& \times\left(-\frac{\exp \{2 \lambda \xi\}-\exp \{\kappa \xi\}}{2(4 i-j)}-\frac{\exp \left\{2 \lambda^{*} \xi\right\}-\exp \{\kappa \xi\}}{2(-4 i-j)}\right. \\
& -\frac{1}{2 s-j}\left(2 \xi \sqrt{|\boldsymbol{k} \cdot \boldsymbol{n}|} \exp \left\{\left(\lambda+\lambda^{*}\right) \xi\right\}\right. \\
& \left.\left.+4\left(\exp \left\{\left(\lambda+\lambda^{*}\right) \xi\right\}-\exp \{\kappa \xi\}\right) \frac{s}{2 s-j}\right)\right)
\end{aligned}
$$

where the definition $\kappa=-(1+j s) \sqrt{|\boldsymbol{k} \cdot \boldsymbol{n}|}$ has been introduced. Only the component proportional to $\boldsymbol{k} \times \boldsymbol{r}$ of $\boldsymbol{n} \times \overline{\boldsymbol{u}}_{2}^{B}$ will be needed in the further analysis which is thus 
given explicitly in the following expression:

$$
\begin{aligned}
\boldsymbol{n} \times \overline{\boldsymbol{u}}_{2}^{B}= & \boldsymbol{n} \times(\boldsymbol{k} \times \boldsymbol{r})(\ldots)+\boldsymbol{k} \times \boldsymbol{r} \frac{|\boldsymbol{n} \times \boldsymbol{k}|^{2}}{8|\boldsymbol{k} \cdot \boldsymbol{n}|^{2}}\left(\left(\left(\exp \{2 \lambda \xi\}-\exp \left\{2 \lambda^{*} \xi\right\}\right) / i-(\exp \{\kappa \xi\}\right.\right. \\
& \left.\left.-\exp \left\{\kappa^{*} \xi\right\}\right) / j\right) / 12+\frac{2 \xi \sqrt{|\boldsymbol{k} \cdot \boldsymbol{n}|}}{5 s} \exp \left\{\left(\lambda+\lambda^{*}\right) \xi\right\} \\
& +\left(\frac{17}{5}+\frac{4|\boldsymbol{k} \cdot \boldsymbol{n}|^{2}}{|\boldsymbol{n} \times \boldsymbol{k}|^{2}}\right) \frac{s}{20}\left(2 \exp \left\{\left(\lambda+\lambda^{*}\right) \xi\right\}-\exp \{\kappa \xi\}-\exp \left\{\kappa^{*} \xi\right\}\right) \\
& \left.+\left(\frac{3}{50}-\frac{4|\boldsymbol{k} \cdot \boldsymbol{n}|^{2}}{10|\boldsymbol{n} \times \boldsymbol{k}|^{2}}\right)\left(\exp \{\kappa \xi\}-\exp \left\{\kappa^{*} \xi\right\}\right) / j\right) .
\end{aligned}
$$

The integration of this expression over $\xi$ yields

$$
\int_{0}^{\infty} \boldsymbol{n} \times \overline{\boldsymbol{u}}_{2}^{B} \mathrm{~d} \xi=\boldsymbol{n} \times(\boldsymbol{k} \times \boldsymbol{r}) G(\boldsymbol{k} \cdot \boldsymbol{n})+\boldsymbol{k} \times \boldsymbol{r} \frac{s}{\sqrt{|\boldsymbol{k} \cdot \boldsymbol{n}|}}\left(\frac{49|\boldsymbol{n} \times \boldsymbol{k}|^{2}}{4800|\boldsymbol{k} \cdot \boldsymbol{n}|^{2}}+\frac{1}{20}\right),
$$

where again the function $G$ has not been given explicitly since it will not be needed in the analysis of $\S 4$.

\section{REFERENCES}

AldRIDGE, K. D. 1967 An experimental study of axisymmetric inertial oscillations of a rotating liquid sphere. PhD thesis, Massachusetts Institute of Technology, Cambridge, MA.

Aldridge, K. D. \& Toomre, A. 1969 Axisymmetric inertial oscillations of a fluid in a rotating spherical container. J. Fluid Mech. 37, 307-323.

Greenspan, H. P. 1968 The Theory of Rotating Fluids. Cambridge University Press.

Greenspan, H. P. \& Weinbaum, S. 1965 On nonlinear spin-up of a rotating Fluid. J. Math. Phys. 44, 66-85.

Noir, J., Hemmerlin, F., Wicht, J., Baca, S. M. \& Aurnou, J. M. 2009 An experimental and numerical study of librationally driven flow in planetary cores and subsurface oceans. Phys. Earth Planet. Inter. 173, 141-152.

Stewartson, K. \& Roberts, P. H. 1963 On the motion of a liquid in a spheroidal cavity of a precessing rigid body. J. Fluid Mech. 17, 1-20.

Van Hoolst, T., Rambaux, N. \& Karatekin, Ö. 2009 The effect of gravitational and pressure torques on Titan's length of day variations. Icarus 200 (1), 256-264.

WAHr, J. M. 1988 The earth's rotation. Annu. Rev. Earth Planet. Sci. 16, 321-349. 